\title{
Development and validation of a digital image processing- based pill detection tool for an oral medication self-monitoring system
}

\author{
Jannis Holtkötter 1,2, Rita Amaral 1,2,3,4, Rute Almeida 1,2, Cristina Jácome 1,2, Ricardo Cardoso ${ }^{5}$, Ana Pereira ${ }^{1,2}$, \\ Mariana Pereira ${ }^{1,2,5}$, Ki H. Chon ${ }^{6}$, João Almeida Fonseca 1,2,5
}

1 MEDCIDS - Department of Community Medicine, Health Informatics and Decision, Faculty of Medicine, University of Porto, 4200-450 Porto, Portugal

2 CINTESIS - Center for Health Technology and Services Research, Faculty of Medicine, University of Porto, 4200-450 Porto, Portugal

3 Department of Cardiovascular and Respiratory Sciences, Porto Health School, Porto, Portugal

4 Department of Women's and Children's Health, Paediatric Research, Uppsala University, Uppsala, Sweden

5 MEDIDA-Serviços em Medicina, Educação, Investigação, Desenvolvimento e Avaliação, LDA, 4200-386 Porto, Portugal

6 Department of Biomedical Engineering, University of Connecticut, Storrs, CT 06269, USA

* Correspondence: fonseca.ja@gmail.com

\begin{abstract}
Objective tools to track medication adherence are lacking. A tool to monitor pill intake that can be implemented in mHealth apps without the need for additional devices was developed. We propose a pill intake detection tool that uses digital image processing to analyze images of a blister to detect the presence of pills. The tool uses the circular Hough transform as a feature extraction technique and is therefore primarily useful for the detection of pills with a round shape. This pill detection tool is composed of two steps. First, the registration of a full blister and storing of reference values in a local database. Second, the detection and classification of taken and remaining pills in similar blisters, to determine the actual number of untaken pills. In the registration of round pills in full blisters, $100 \%$ of pills in gray blisters or blisters with a transparent cover were successfully detected. In counting of untaken pills in partially opened blisters, $95.2 \%$ of remaining and $95.1 \%$ of taken pills were detected in gray blisters, while $88.2 \%$ of remaining and $80.8 \%$ of taken pills were detected in blisters with a transparent cover. The proposed tool provides promising results for the detection of round pills. However, the classification of taken and remaining pills need to be further improved, in particular for the detection of pills with non-oval shapes.
\end{abstract}

Keywords: computer vision; image processing; medication adherence; object detection; pill detection

\section{Introduction}

Worldwide, in 2019, patients collectively took medications on 1.8 trillion days, with a 3\% annual increase since 2014. Pills are one of the most commonly prescribed formulations and one of the most preferred approaches by patients [1]. The preferred way of distributing prescribed medication in Europe $(80 \%)$ is in the form of blister packs. Calendarized blister packs may improve compliance to prescribed medication by counteracting nonadherence factors, such as unintentional forgetfulness [2], [3]. Moreover, blister packs allow third parties, for example caregivers, to monitor the removal of the medication of the blister pack.

A crucial aspect for successful treatment is the patient adherence to prescribed medications. Poor adherence can diminish the effectiveness of the treatment plan and lead to severe risks for the patient. Yet, it is known that the adherence rate in patients with chronic 
diseases is low and approximately $50 \%$ of patients in developed countries do not take their medications as prescribed [4].

Assessment of adherence is an important aspect for a precise evaluation and adjustment of medical treatments. However, the measurement of adherence in the community is a difficult challenge since a "gold standard" method does not exist. Commonly employed subjective methods for adherence monitoring are either physician- or self-assessment, and pill counting. These methods are easy to implement and use, not very costintensive, and can achieve good results. However, they have also been shown to be imprecise and heavily dependent on the collaboration between patient and healthcare provider [5], [6], [7].

Electronic monitoring devices (EMDs) are a more objective method of adherence monitoring. Despite being proven to be highly accurate, the use of EMDs is limited in large populations due to high costs and possible practical issues, such as difficulties during the medication refilling in a local pharmacy [9]. Another straightforward and low-cost method that can be applied to different types of medication, such as tablets or inhalers, is dose counting, in which the number of doses taken is compared to the number of doses the patient has received, at the end of the analysis period. However, it only measures the average adherence and lacks information about daily adherence and other patterns of medication usage [8].

With the knowledge that nonadherence is a major concern in chronic care and that current methods for adherence monitoring are biased, imprecise or costly and difficult to implement, new reliable methods, easy to implement and inexpensive, are needed. Smartphones accompany us in everyday life and therefore many applications, including for support of medication adherence, are being developed [9].

A typical modern smartphone is equipped with imaging sensors that can produce images of exceptional resolution and quality. Digital image processing has already played an important role in several projects related to the detection or identification of pills [10][12]. Lee and co-workers proposed an automatic pill identification and recognition program to identify illegal drugs [10]. In their proposal, an image of a pill is processed in several stages including preprocessing, edge detection, and feature vector construction. The extracted feature vectors are then compared to an existing pill database to create a ranking of pills that are most likely to match. Another mobile solution was proposed by Cunha et al. [11]. Their tool, HelpmePills, aims to support elderly people to prevent wrong ingestion by creating a personalized database of their medication history. The user is asked to place a pill on a detectable marker square and capture an image which is then automatically processed to extract information such as the shape, dimensions, and color of the pill. After creating a local database, the user can take a picture of the pill before the intake and the tool will compare it with the database for identification. The aforementioned pill identification tools require an initial database of pill features to correctly identify them. A project with the main focus on the development of an image processing software able to identify broken and unused pills in a blister pack for medication quality monitoring at production facilities was proposed by Rani et al. [12]. During a preprocessing stage, irrelevant details of the image, such as background and noise, are removed or at least reduced. Moreover, digital filters are applied to the image to highlight maximum intensity levels and detect edges that are created by the boundaries of the pills. The detected edges are converted into a binary image consisting of only the black and white pixels, in which ideally only the pill boundaries are displayed by white pixels. The binary image is then further analyzed for gaps between the detected edges using a horizontal and vertical histogram. These gaps should indicate the beginning and end of each individual pill boundary and therefore segment the image. Each individual pill segment is then used to extract features from it, which indicate if the pill is damaged, half-filled, or undamaged. The feature extraction is performed by correlation features, which describe the variation among the appearance of images of one type to another. Very encouraging experimental results of $96.33 \%$ accuracy were achieved over 50 blisters. Nevertheless, no image-based solutions to measure medication adherence were found. 
The objective of this research was to develop a pill detection tool able to count the number of remaining pills in a blister pack. The tool is intended to use images captured by smartphones in real-world conditions and to be compatible with as many pill blister types and shapes as possible, without the need to predefine a database for each blister brand.

The tool was designed with the possibility of integration in mobile health solutions including INSPIRERS-HTN [13], for the management of hypertension, and AIRDOC [14] or InspirerMundi [15], for supporting self-management of chronic respiratory diseases.

In this paper, we i) present the pipeline of the proposed pill detection tool, including the used image processing techniques, and ii) present and discuss the results of its experimental validation.

\section{System Overview}

To cope with the goal to design a system compatible with variable pill blister types, we decided to consider a self-configuration approach. An initial image of the full blister will be used to extract a blister-specific configuration using the pill detection tool. Then, posterior images of the same or equal blisters are processed with respect to that configuration. Medication adherence can be estimated from successive detection of missing pills from the series of blister images.

The pill detection tool consists of three basic phases: image acquisition, image preprocessing, and detection and counting, as displayed in Figure 1. This prototype was developed with MATLAB software (The MathWorks Inc., Natick, MA, USA) on a computer for rapid development.

\section{A. Image acquisition}

The first phase starts with the loading of an image of a pill blister into the pill detection tool. In the current offline MATLAB version, an image acquired using the smartphone camera is loaded for processing. Once implemented in a smartphone application, the user would have the possibility to either take an image with the smartphone camera or use a previously captured image from the image library. After the image is read in, it needs to be cropped in order to remove as much background from the image as possible and retain only the region of the blister. Ideally, the blister image is captured on a plain and flat background for a quick automatic blister detection to succeed.

First, the image is converted into grayscale and filtered by a median filter with a neighborhood size of 5 by 5 pixels, which reduce noise and smooth the image. Then, the image is further filtered by a local standard deviation filter with a neighborhood size of 9 by 9 pixels to highlight contours and edges in the image. By using a global threshold technique, the image is then transformed into a binary image, which displays heterogeneous areas as white objects, and homogeneous areas as the black background, as illustrated in the top row of Figure 1. 


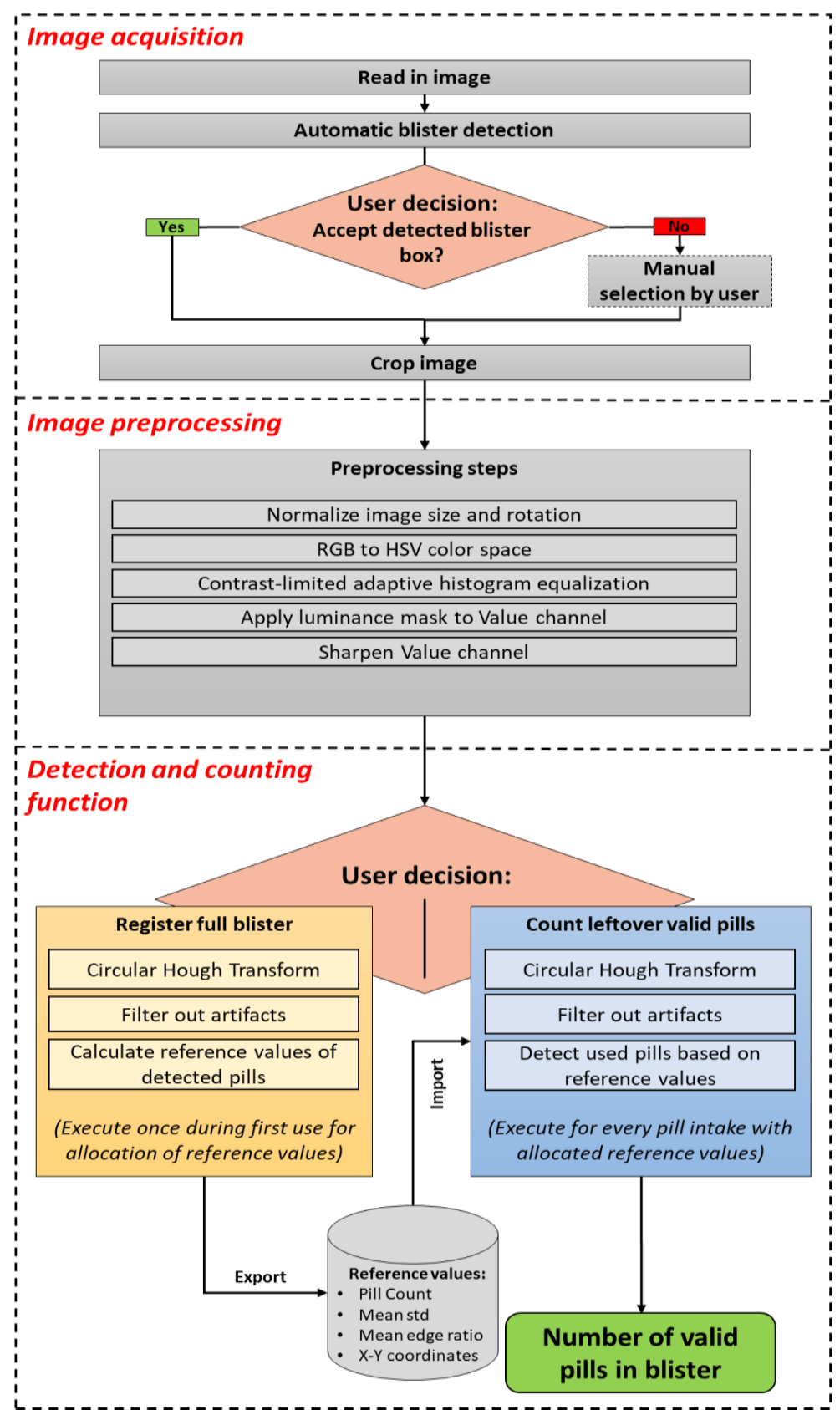

Figure 1. Pill detection tool pipeline

Most binary images at this step will contain several individual connected components, which often originate from the various contours on the blister. Furthermore, it is assumed that although the contours of the blisters are represented as individual connected components, they do not necessarily represent one single connected blister object. Moreover, there can also be objects in the background and general artifacts that disturb a homogeneous background of the binary image. To connect individual objects that have proximity to each other and to filter out possible confounding factors, morphological operations are employed as two consecutive dilations with a horizontal and vertical line of 50 pixels as a structural element, followed by a closing operation with a 50 by 50 pixels square as a structural element. Finally, the largest connected component in the image is chosen as the blister, since smaller objects or artifacts in the background could still be present after the initial morphological operations. The smallest enclosing rectangle, called bounding box, is then proposed to the user as the blister. The progress of the morphological operations and the detected bounding box is displayed in the second row of Figure 2. 


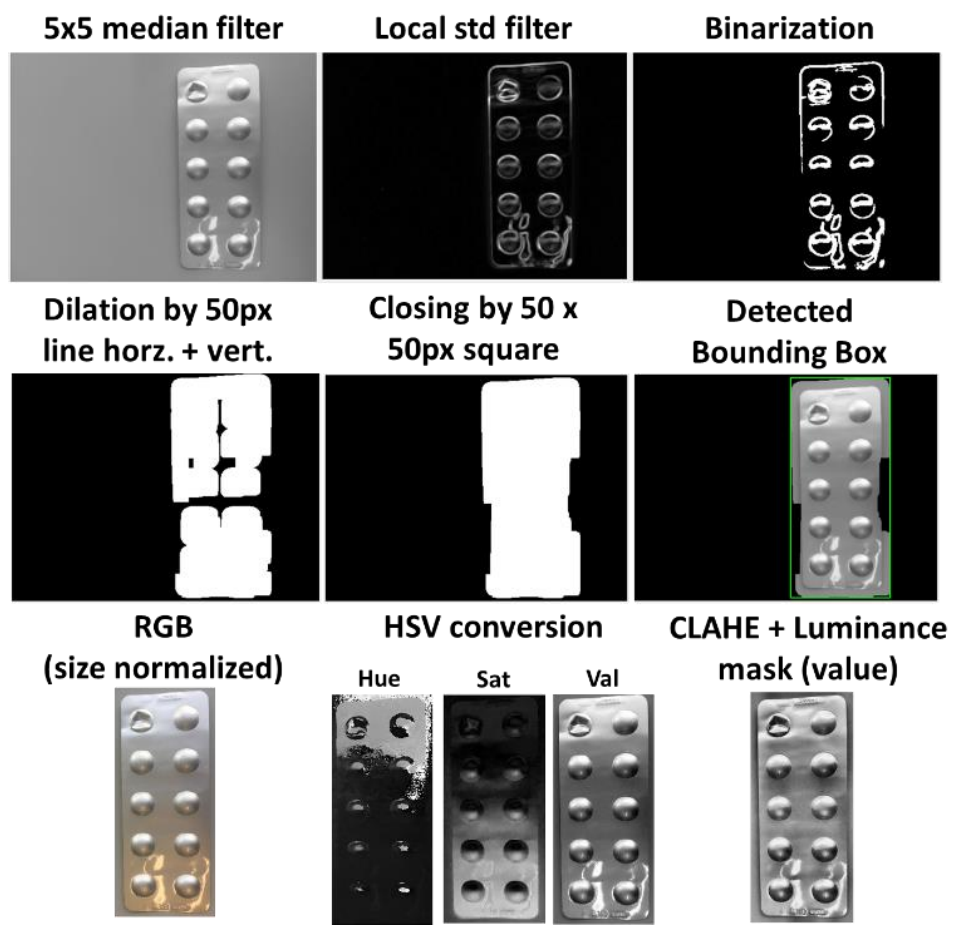

Figure 2. Individual steps of automatic blister detection and image preprocessing

The user can either confirm or refute the selected region. If the selected region is refuted, the user must manually slide an adjustable rectangle over the blister. After automatic or manual selection of the blister region, the image is cropped by that region to be used in the further steps.

\section{B. Image preprocessing}

The cropped image is then preprocessed to ensure that it has the required quality and comparability between multiple images of the same blister. First, the image size is scaled to 1,000 pixels at the long edge while keeping the relation to the short edge. Additionally, the image is rotated as needed so that the blister is always vertically aligned. After this normalization, the image is converted from RGB (Red, Green, Blue) into the HSV (Hue, Saturation, Value) color space. Then, each channel is enhanced by contrast limited adaptive histogram equalization (CLAHE) to improve the local contrast of the images and enhance details' definition, such as edges. The value channel plays an important role in the detection of edges in further processing functions. Thus, a luminance mask is applied to this channel to remove possible reflections from the image capturing process, which could distort the pixel intensities in the processing. Therefore, a region filling function based on inward interpolation is performed on all pixels over an intensity value of 0.95 . Finally, the value channel is sharpened by a radius of 6 pixels and an strength of 60 to increase the detectability of edges and details in the image. The image preprocessing steps are illustrated in the bottom row of Figure 2.

\section{Detection and counting}

The main step, which is responsible for counting the number of observed pills in the blister, consists of two separated branches: the registration and the counting modes. The basic concept of both branches is similar, however certain steps are executed differently. The registration mode is designed to detect all pills in full blisters from which no pill was dispensed yet, with no previous information available, and to retrieve reference values for that blister at the best possible conditions. The counting mode intends to detect and count remaining valid pills in a potentially (partially) used blister from the previously obtained reference values for that blister form at registration mode. Once the reference values for a type of blister are allocated, the counting mode can also be used to detect the number of pills in a full blister without allocating new reference values. 
The registration mode begins with the detection of edges in the preprocessed value channel of the image, using the Canny edge detector [16]. This produces a binary image in which all edge classified pixels are displayed as white pixels and the rest as black pixels. After this edge detection, it is possible to detect circular shapes in the image with the use of the Circle Hough Transform (CHT). The CHT is a basic feature extraction function that uses a voting system in the Hough parameter space and the selection of local maxima in an accumulator matrix to detect circles [17]. For each detected circle, the radius, the center coordinates, and the metric value are noted. To mainly include actual pills only circles with a radius between 30 and 130 pixels are kept. The metric value ranges between 0 and 1 , on which 1 expresses a high certainty of the detected object being circular. The sensitivity of the CHT was set by trial and error to 0.93 , balancing between the inclusion of all pills and artifacts reduction.

It should be noted that round blister corners, structures on the blister, and reflections can introduce artifacts. Assuming that most detected circles are actual pills, as a full blister should be considered for registration mode, a combination of mean-based rules is used to discard false detections. The employed metric properties to reduce false detection consisted of the circle radius, metric value, deviation from median $\mathrm{x}$-axis position, and the ratio of white pixels to a total number of pixels per circle, which we will refer to as "circle edge ratio". These properties are analyzed and compared to their mean values for each detected circle in several conditional statements to determine outliers.

First, circles with a radius deviating more than $50 \%$ from the mean radius are classified as artifacts. Following, the circle edge ratio is used to further classify the remaining circles. To react dynamically on the appearance of the Canny edge detected image, the standard deviation of the circle edge ratio across all circles is calculated. A threshold of 0.04 is chosen to differentiate between two cases: high or low standard deviation of circle edge ratio. For the high case, the standard deviation is included in the upper and lower limit of the acceptable circle edge ratio. In case of low standard deviation, the value itself is not used in the classification process because it tends to remove pills in images which have no more artifacts at this point. In both cases, high or low standard deviation, the metric value is considered if the mean is over 0.15 , which indicates a certain informative value. A low metric mean value of under 0.15 shows that the metric value is not a good indicator to differentiate actual pills from artifacts. In case of a sufficient mean metric value all circles with a metric value above $75 \%$ of the metric median are classified as pills and not as artifacts, even if the circle edge ratio indicates otherwise. This action should prevent an incorrect rejection of pills due to reflections or slight deformations of the package. Finally, all remaining circles are checked for their $x$-axis position in relation to the median $x$-axis position for left and right half of the blister pack. Due to vertical rotation during the preprocessing steps the pills in the blister should be in vertical columns. For the columns at most right and left sides of the blister, every pill that deviates from the median $x$-axis position by more than $5 \%$ of the image width is further checked regarding deviation distance, circle edge ratio, and metric value. The deviation limits for circle edge ratio and metric value are stricter compared to the previous classification step. All identified outliers are considered as artifacts and discarded. All remaining circles are identified as pills.

A binary mask of the reference identified pills is then created based on the centercoordinates and radii of the classified pills, as illustrated in the two examples of a successful classification in Figure 3.

The final step of the registration mode is the calculation of reference pill values: mean radius, mean circle edge ratio, center-coordinates, and mean local standard deviation of all pills. The local standard deviation based on the pixel neighborhood represents the heterogeneity of pixel intensities in different regions. The mean of the local standard deviation for pixels within detected circles serves as a reference for detecting a pill. 


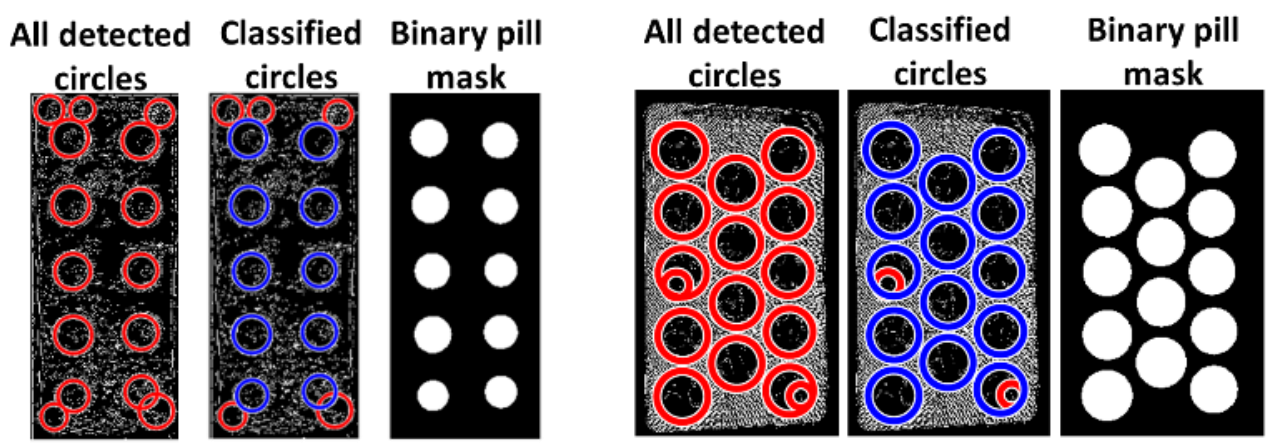

Figure 3. Two examples of the pill mask after classification of circles

The counting mode aims to detect and count the number of pills in an image of a blister that can be already partially used. Again, all circular shapes in the image are detected by a CHT and need to be then further classified into either an artifact or a detected pill. However, since empty pill pockets from taken pills are often detected as circular shapes, the mean property values, as used in the registration mode are not sufficient as comparison values for a pill that is present. Therefore, the detected circles are compared to the previously obtained reference values of the corresponding full blister.

First, for each position of the reference pills the closest circle detected in the current image is determined by comparing the center coordinates. Considering a proximity as the mean pill radius plus 10 pixels, if more than one circle candidate is detected, the circles with the lowest deviation from the reference mean radius is chosen. This approach allows corresponding detected circles from registration to the ones in the opened blister, which originated from either a valid pill or from the empty pill pocket.

The comparison of coordinates assumes a certain level of similarity between current image and reference image regarding image crop. Blisters with an odd number of pills often have an asymmetrical pill alignment. The method of comparing the center coordinates from reference to the new image could produce misclassifications if the user rotated the blister by $180^{\circ}$ concerning the image of the full blister. Therefore, if the full blister carries an odd number of pills, an additional step is added. For this, a copy of the preprocessed image is produced, rotated by $180^{\circ}$ and the counting mode is applied. For each image version, original and rotated, the distance of each closest circle to the reference pills is added up and the version with the smaller total distance is selected for further use.

After the identification of the closest circles to the reference pills, the local standard deviation and the circle edge ratio of each circle are compared to the respective reference values. First, the local standard deviation is checked. An increase of 0.05 compared to the reference value leads to classification as a used pill. If the increase is lower than 0.05 but higher than 0.02 the circle edge ratio is consulted. If it shows an increase of at least 0.025 the pill in also classified as already used. These two properties are chosen because empty pockets should lead to an increase in the local standard deviation due to the higher heterogeneity of the surface and the deformations of the used pill pockets change the circle edge ratio. The appearance of these intensities in a blister with damaged and undamaged pill pockets is displayed in Figure 4. The remaining circles are labeled as present pills and used as the final count number.

Since the detection and counting mode is implemented in a two-step approach to provide self-configuration, the user must register a new blister at least once before the pill counting mode can be utilized. Important parameters are detected and stored in the registration step, which is required for the successful execution of the pill counting step. 

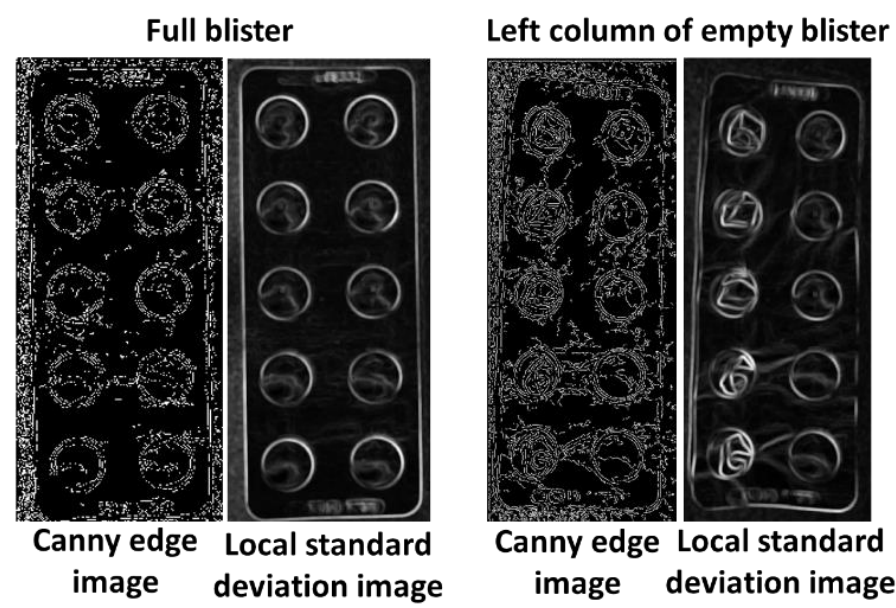

Figure 4. Intensity increase of local standard deviation and circle edge ratio due to deformation of pill pockets

\section{Datasets}

The dataset used during the development phase to determine metrics and thresholds consists of a total of 179 images of 9 different blisters. From those, 103 images display full blisters and were used to develop the registration mode. The other 76 images were used for the development of the counting mode and consist of 8 sequences, containing the full blister and a series of subsequent states in which a variable (not necessarily consecutive) number of pills is missing.

The testing dataset consists of a total of 280 images of 20 different blisters, taken in 31 sequences. Of the 20 different blisters, 9 blisters are same as in the development dataset. Images with full blisters were used as a subset to test the registration mode (31 images). The sequences were used to test the counting mode (249 images).

The images in both datasets contain only blisters with round pill pockets in a variety of pill counts, and pills and blisters of different shapes and colors. The images were captured by several members of the research group with different smartphones in varying environments to simulate the variability of real-world blisters photographs taken by potential app users.

The blisters were categorized into two different types (Figure 5):

- Gray: Blisters of gray color with gray pill cover

- Transparent: Blisters with a transparent pill cover, irrespective of the background color

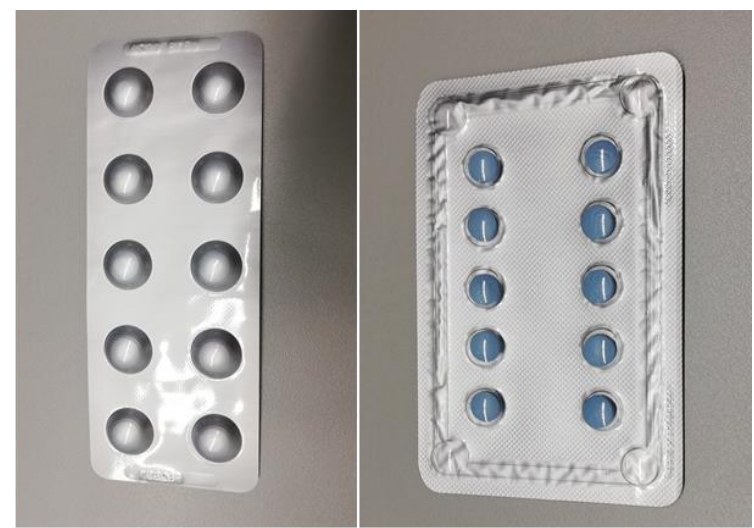

Gray blister type

Transparent blister type

Figure 5. Examples of different blister types used in the datasets

\section{Validation Strategy}


Three experimental validations were performed using varying subsets of the testing dataset. First, the automatic blister detection was tested with 60 images of 14 different, randomly chosen, blister brands. In 30 of these images, the blister was captured on a flat hand and in the other 30 images, the blister was captured in front of a flat, plain background. Second, the registration mode was tested with the full blister reference image from each sequence, including 31 images. Third, the counting mode was tested with the full testing dataset of 249 images in 31 sequences. It should be noted that the 31 reference images that were used in the registration mode to prepare the reference values used in the counting mode, were not included in the validation of the counting mode.

The experimental validation plans are summarized in Table 1.

Table 1. Experimental validation plans

\begin{tabular}{|c|c|c|}
\hline Test & Dataset & Test description \\
\hline $\begin{array}{l}\text { Test } 1 \\
\text { (Automatic blister } \\
\text { detection) }\end{array}$ & $\begin{array}{c}\mathrm{n}=60 \text { images } / 14 \text { different } \\
\text { blisters }\end{array}$ & $\begin{array}{l}\text { Tests on images in different environments and counts how } \\
\text { often the blister detection succeeded (subjective } \\
\text { assessment). }\end{array}$ \\
\hline $\begin{array}{c}\text { Test } 2 \\
\text { (Registration mode) }\end{array}$ & $\begin{array}{c}\mathrm{n}=31 \text { images / } 20 \text { different } \\
\text { blisters / } 323 \text { pills }\end{array}$ & $\begin{array}{l}\text { Tests on images of full blisters and counts how many pills } \\
\text { and false positives are detected. }\end{array}$ \\
\hline $\begin{array}{l}\text { Test } 3 \\
\text { (Counting mode) }\end{array}$ & $\begin{array}{l}\mathrm{n}=249 \text { images } / 20 \text { different } \\
\text { blisters } / 1267 \text { pills }\end{array}$ & $\begin{array}{l}\text { Tests on blisters with a varying number of present pills. } \\
\text { Counts how many present pills are detected, and how } \\
\text { many taken pills are correctly classified. }\end{array}$ \\
\hline
\end{tabular}

\section{Results}

First, the efficacy of the automatic blister detection was tested. Each blister detection was evaluated manually and specified as successful if the detected blister was positioned centered in the region, and the border around the blister did not exceed $1.5 \mathrm{~cm}$. The automatic detection of blisters on a flat surface, like a table, was successful in 24 out of 30 images and failed 6 times due to edges in the background, or objects close to the blister. For blisters on the hand, the detection failed in 28 out of 30 images.

The results of the registration mode test are displayed in Table 2. All pills in both blister types were detected. The number of false positives, in which an artifact is falsely identified as pill, is slightly higher for transparent blisters: $0.4 \%$ compared to $0.2 \%$ in gray blisters.

The summary of the results for the counting mode is displayed in Table 3. The gray blister type showed an accuracy of $95.2 \%$, which is over $10 \%$ higher than the accuracy for the transparent blister type.

Table 2. Results of Test 2 (Registration mode)

\begin{tabular}{|c|c|c|c|}
\hline \multicolumn{4}{|c|}{ Blister type } \\
\hline & Gray & Transparent & Total \\
\hline Number of images & 17 & 14 & 31 \\
\hline Total tested pills & 159 & 164 & 323 \\
\hline Total detected pills & 162 & 170 & 332 \\
\hline False positives & 3 & 6 & 9 \\
\hline Correct pill detections & 159 & 164 & 323 \\
\hline Correct pill detection (\%) & $100 \%$ & $100 \%$ & $100 \%$ \\
\hline False positives $(\%)$ & $0.2 \%$ & $0.4 \%$ & $0.3 \%$ \\
\hline
\end{tabular}




\begin{tabular}{|c|c|c|c|}
\hline & \multicolumn{2}{|c|}{ Blister type } & \multirow[b]{2}{*}{ Total } \\
\hline & Gray & Transparent & \\
\hline Number of images & 130 & 119 & 249 \\
\hline Total present pills tested & 581 & 686 & 1267 \\
\hline Correct present pills detected & 553 & 605 & 1158 \\
\hline Correct present pill detections (\%) & $95.2 \%$ & $88.2 \%$ & $91.4 \%$ \\
\hline Total taken pills tested & 659 & 682 & 1341 \\
\hline Correct taken pills detected & 627 & 551 & 1178 \\
\hline Correct taken pills detections (\%) & $95.1 \%$ & $80.8 \%$ & $87.8 \%$ \\
\hline Accuracy & $95.2 \%$ & $84.5 \%$ & $89.6 \%$ \\
\hline
\end{tabular}

The difference between gray and transparent blister became very apparent in the image version filtered by the Canny edge detector or the local standard deviation (Figure 6). Edges and especially deformations in the area of the pill cover were much more visible in gray blisters due to the stronger contrast differences of the material.

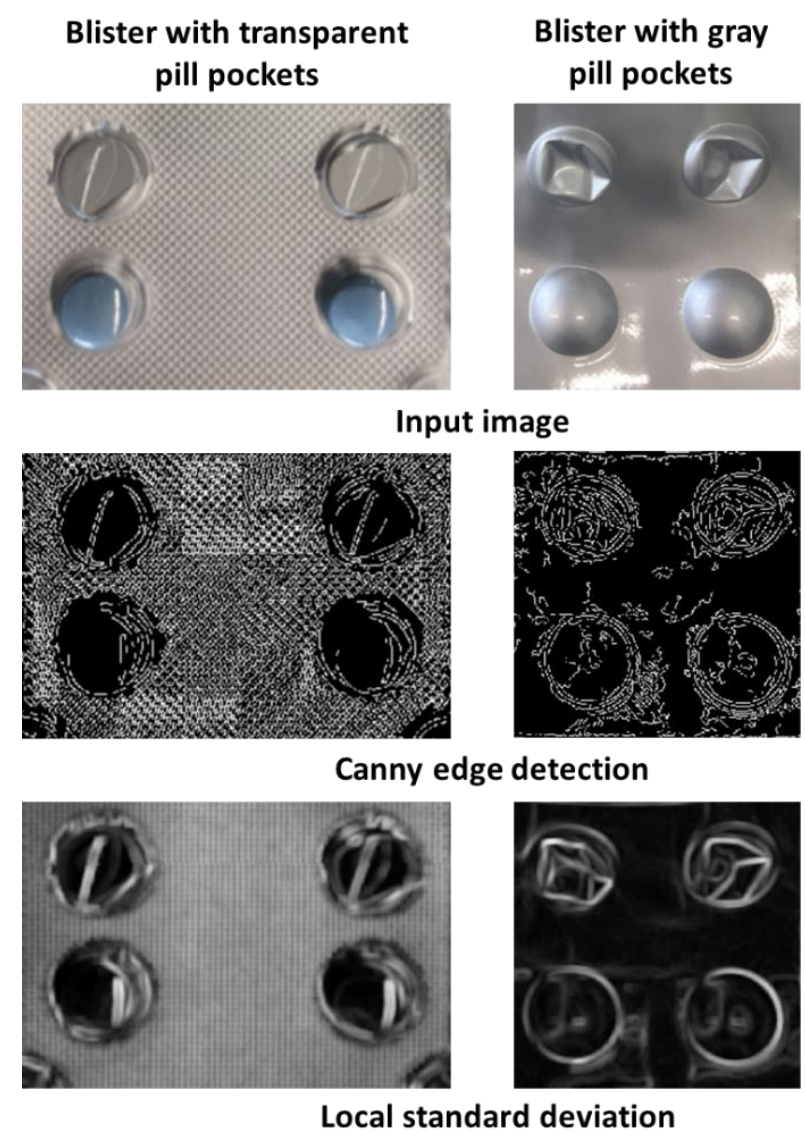

Figure 6. Comparison of transparent and gray pill pockets

\section{Discussion}

We proposed a digital image processing-based pill detection tool, which included function capabilities for automatic blister detection, registration of a full blister, and counting of present and missing pills in blisters that can be already partially used. The use of CHT in the registration and counting mode enabled accurate detection of pills in blisters with round pills. Keeping this in mind the dataset for the experimental validation was composed only of blisters with round pills. For the registration mode very good results, with almost perfect detection, were achieved. Good results were also achieved for the 
counting mode and our approach was better for blisters that cover the pill with a gray, opaque material than for those with a transparent pill cover. The results are promising and, although there is a need for additional improvement, this tool has potential to be further developed and adapted for the modular implementation in future mHealth applications.

The automatic blister detection is intended to improve experience since it requires less user input. The automatic blister detection showed acceptable results when the blister is placed on a flat surface like a table ( $80 \%$ successfully detected). The detection of the blister on the hand mainly fails due to the assortment of details and structures in the background. Therefore, the future recommendation for the user should be to place the blister on a table or other flat and homogeneous background. Since this is a simple instruction and the fulfillment of these requirements is relatively easy to comply with, this automatic blister detection tool is potentially useful to be used as the first step in pill detection. Nevertheless, it is also possible to include a functionality allowing the user to reject the automatic detection and use a manual cropping instead.

The experimental validation tests of the registration mode showed a correct pill detection rate of $100 \%$ for round pills in blisters of gray or transparent type. In addition, the low rate of false-positive detections $(0.3 \%$ in total) indicates high accuracy for the detection of round pills, as expected due to the use of CHT. The small number of false positives can be allowed to be removed by the user in an additional verification step final to the registration of the blister. Moreover, the testing dataset consisted of only 31 images and needs to be further extended.

An important outcome of the registration mode is the precise location of the pills within the blister, since this is an important asset for the extraction of pill features. There was the risk that too many artifacts would cause the mean property values to be skewed and therefore not give an estimation of the actual pill properties. Skewed mean property values would cause misclassification of the pills and artifacts. However, the results of the registration mode test for the gray and transparent blister show that a skewing of the mean property values was not an issue in the test dataset.

The counting mode used the values that were calculated for the individual pill regions with the registration step. It was able to detect 1158 out of 1267 circular pills (91.4\%) present in the blisters. This result is not far from the results reported by Rani et al. [12] on 15 blisters with circular pills: 125 pills detected out of 131 present (95.4\%). Moreover, the automated defective tablet detection tool from Rani et al is adapted to the production process in pharmaceutical industries, where the image conditions are much more controlled and predictable compared to the image acquisition with a smartphone, regarding light/reflections, blister alignment in the image and presence of artifacts. Also, false positive detections are not relevant for inspection and were not reported. Due to the general lack of literature regarding pill detection in blister images acquired by smartphones in near realworld environments, an extensive comparison of the proposed results is limited.

The accuracy of the counting mode was higher for blisters that were covered by a gray, opaque material, than for blisters with a transparent pill cover. For the differentiation between damaged and valid pill pockets, values that are dependent on the heterogeneity and the visibility of edges were used. In blisters with gray pill pockets deformations create clear edges with several strong contrast changes compared to undamaged pills. These edges become clearer after filtering by the Canny edge detector and local standard deviation. However, blisters with transparent pill pockets pose a difficult challenge for the detection of taken pills. Due to the transparent material deformations were more difficult to detect since edges and contrast changes were less visible. Therefore, the intensity increase for damaged pills is not that obvious in the filtered versions of the image and ultimately the damaged pill pockets remain undetected.

A possible solution to improve the detection of pill presence with transparent cover could be the usage of different image channels, such as hue or saturation channel that create an image version based on the pixels colors or saturation. Since the pills are visible behind the transparent pill cover, the missing of a pill should show more clearly. 
However, an empty pill pocket might still appear to be filled if the blister was placed on a surface of the same color as the pills and the background thereby optically replaces as the missing pill. Incorporating pill color information during registration mode could allow to distinguish pills and backgrounds with different colors Nevertheless, this would require information on the type of blister (transparent vs gray), either extending the amount of necessary user input or requiring an automatic method of determining the blister type.

Reflections, deformations of a valid pill slot, unsharp images, or objects in front of the blister, also caused misdetections. Reflections on the surface of the pills were influenced by several factors such as the lighting conditions of the image capture environment, use of flash, and the material of the blister. Reflections on the pills can be interpreted as edges or deformations due to the induced contrast heterogeneity in the pill region. Therefore, valid pills with strong reflections can be misclassified as "taken". The same issue arose for detection of valid pills when deformations of the pill pocket were present. If the deformations caused an increase in the intensity of the local standard deviation and canny edge detected version of the image, it was likely that the same pill was misclassified as taken in every image of a blister sequence. Further, unsharp images usually produced multiple misdetections since the pill regions were not precise, and deformed pill pockets showed no clear difference to valid pill pockets because edges were blurred. Finally, another source of misdetection was the presence of interfering objects, such as a finger, that overlapped one or several pills in the images. The outline of the interfering objects in the region of the pill led to the misclassification of present pills as taken.

In general, the most important limitations and challenges of the proposed pill detection tool were the correct detection of valid and taken pills in blisters with transparent pill cover and the correction of several sources of misdetection.

An approach for future improvement of the pill detection tool should include a further tuning of the reference values which are used to differentiate between taken and valid pills. The current reference values are static and only based on the mean of the reference image. Defining dynamic value ranges that rely on the overall change in intensity values within the image could improve the performance of the detection when changes in the image capture conditions occur. In addition, further transformations of the image should be tested to search for alternative metrics that are able to differentiate more clearly between valid and taken pills. Considering the specific purpose of adherence quantification, a further way to improve the pill detection in a series of blisters could be the incorporation of data from previous detections of the same blister, complementing information obtained from the reference image. This could include the updated number and positions of pills from previous images or even the number of pills expected to be present according to the treatment plan.

Furthermore, different approaches to the problem of pill detection and counting could be considered. For example, machine learning methods can be explored in their ability to classify pocket pills as full or empty. However, it must be realized that the training of machine learning models requires large datasets that are composed of a wide variety of various types of blisters, requiring a much larger database.

Additionally, this pill detection tool needs to be extended to accommodate a detection mode for oblong pills. A base for this could be the automated tablet recognition tool, presented by Rani et al. [12], in which the defective blisters are identified using correlation features that describe the variation among the appearances of the individual pills to each other, not assuming a particular shape contour, as does the CHT.

\section{Conclusion}

Our tool provides a well performing pill detection for blister images captured by a smartphone in a near real-world environment. It requires the registration of a full blister and, afterwards, uses extracted information for the counting of present pills in opened blisters. Despite the fact that only blisters with round pill shapes were covered by our tool, 
it provided a useful construct for further development and extension of functions, aiming at a future implementation in smartphone applications.

Author Contributions: Conceptualization, J.H., R.C., R.Am., R.Al. and J.A.F.; coding, J.H., R.C., R.Al.; methodology, J.H., R.Am, R.Al, J.A.F.; investigation, J.H., R.Al, R.C., C.J., M.P., A.M.P.; writing - original draft preparation, J.H.; writing - review and editing, all authors; supervision, R.Am.; funding acquisition, J.A.F. All authors have read and agreed to the published version of the manuscript.

Funding: This work was supported by POCI-01-0145-36 FEDER-029130 (“mINSPIRE-mHealth to measure and improve adherence to medication in chronic obstructive respiratory diseases - generalisation and evaluation of gamification, peer support and advanced image processing technologies") through the European Regional Development Fund (ERDF), cofunded by the COMPETE2020 (Programa Operacional Competitividade e Internacionalização), Portugal 2020 and by Portuguese Funds through Fundação para a Ciência e a Tecnologia. and also, by project NORTE-01-0247FEDER-033275 (AIRDOC: Smart Mobile Application for Individualized Support and Monitoring of the Respiratory Function and Sounds of Chronic Obstructive Patients) financed by the North Portugal Regional Operational Programme (NORTE 2020), under the PORTUGAL 2020 Partnership Agreement, and through the European Regional Development Fund (ERDF). AIRDOC is with ITEA 316040 PHE - Personal Health Empowerment project consortium.

Institutional Review Board Statement: Not applicable.

Data Availability Statement: The data presented in this study are available on request from the corresponding author.

Conflicts of Interest: The authors declare no conflict of interest. The funders had no role in the design of the study; in the collection, analyses, or interpretation of data; in the writing of the manuscript, or in the decision to publish the results.

\section{References}

[1] D. Denesh, J. Carbonell, J. S. Kane, D. Gracie, and C. P. Selinger, "Patients with inflammatory bowel disease (IBD) prefer oral tablets over other modes of medicine administration," https://doi.org/10.1080/17474124.2021.1898944, 2021, doi: 10.1080/17474124.2021.1898944.

[2] B. K. Zedler, A. Joyce, L. Murrelle, P. Kakad, and S. E. Harpe, "A Pharmacoepidemiologic analysis of the impact of calendar packaging on adherence to self-administered medications for long-term use," Clin. Ther., vol. 33, no. 5, pp. 581-597, May 2011, doi: 10.1016/j.clinthera.2011.04.020.

[3] B. K. Zedler, P. Kakad, S. Colilla, L. Murrelle, and N. R. Shah, "Does Packaging with a Calendar Feature Improve Adherence to Self-Administered Medication for Long-Term Use? A Systematic Review," Clin. Ther., vol. 33, no. 1, pp. 62-73, Jan. 2011, doi: 10.1016/j.clinthera.2011.02.003.

[4] E. Sabate, O. mondiale de la santé, E. Sabaté, and W. H. Organization, Adherence to Long-term Therapies: Evidence for Action. World Health Organization, 2003.

[5] J. Lee et al., "Nonadherence in the era of severe asthma biologics and thermoplasty," European Respiratory Journal, vol. 51, no. 4. European Respiratory Society, Apr. 01, 2018, doi: 10.1183/13993003.01836-2017.

[6] E. Kreys, "Measurements of Medication Adherence_ In Search of a Gold Standard _ Journal of Clinical Pathways," J Clin Pathways, vol. 2, no. $8 . \quad$ pp. 43-47, 2016, Accessed: Dec. 01, 2020. [Online]. Available: https://www.journalofclinicalpathways.com/article/measurements-medication-adherence-search-gold-standard.

[7] C. Jácome et al., "Patient-physician discordance in assessment of adherence to inhaled controller medication: A crosssectional analysis of two cohorts," BMJ Open, vol. 9, no. 11, p. e031732, Nov. 2019, doi: 10.1136/bmjopen-2019-031732.

[8] L. A. Anghel, A. M. Farcas, and R. N. Oprean, "An overview of the common methods used to measure treatment adherence," Med. Pharm. Reports, vol. 92, no. 2, pp. 117-122, 2019, doi: 10.15386/mpr-1201.

[9] Y. Peng et al., "Effectiveness of mobile applications on medication adherence in adults with chronic diseases: A systematic review and meta-analysis," Journal of Managed Care and Specialty Pharmacy, vol. 26, no. 4. Academy of Managed Care 
Pharmacy (AMCP), pp. 550-561, Apr. 01, 2020, doi: 10.18553/jmcp.2020.26.4.550.

[10] Y.-B. Lee, U. Park, and A. K. Jain, “PILL-ID: Matching and Retrieval of Drug Pill Imprint Images," 2010, doi: 10.1109/ICPR.2010.645.

[11] A. Cunha, T. Adão, and P. Trigueiros, “HelpmePills: A Mobile Pill Recognition Tool for Elderly Persons,” Procedia Technol., vol. 16, pp. 1523-1532, 2014, doi: 10.1016/j.protcy.2014.10.174.

[12] N. Shobha Rani, V. K. Nithusha, and T. P. Roshna, “Automatic recognition and verification of defective tablet blisters using entropy based filtering and histogram processing," Int. J. Appl. Eng. Res., vol. 10, no. 5, pp. 13155-13168, 2015.

[13] L. Nogueira-Silva et al., “DEVELOPMENT OF A MOBILE HEALTH APP FOR THE MANAGEMENT OF HYPERTENSION, INCLUDING TREATMENT ADHERENCE ASSESSMENT, USING IMAGE DETECTION TECHNOLOGY - INSPIRERSHTN," J. Hypertens., vol. 39, no. Supplement 1, p. e380, Apr. 2021, doi: 10.1097/01.hjh.0000748952.19902.f5.

[14] R. Almeida et al., AIRDOC: SMART MOBILE APPLICATIONFOR INDIVIDUALIZED SUPPORT AND MONITORINGOF RESPIRATORY FUNCTION AND SOUNDSOF PATIENTS WITH CHRONIC OBSTRUCTIVE DISEASE. 2020.

[15] P. Vieira-Marques et al., "InspirerMundi-Remote Monitoring of Inhaled Medication Adherence through Objective Verification Based on Combined Image Processing Techniques," Methods Inf. Med., vol. 60, no. S 01, pp. e9-e19, Apr. 2021, doi: 10.1055/s-0041-1726277.

[16] J. Canny, “A Computational Approach to Edge Detection,” IEEE Trans. Pattern Anal. Mach. Intell., vol. PAMI-8, no. 6, pp. 679-698, 1986, doi: 10.1109/TPAMI.1986.4767851.

[17] J. Illingworth and J. Kittler, “The Adaptive Hough Transform,” IEEE Trans. Pattern Anal. Mach. Intell., vol. PAMI-9, no. 5, pp. 690-698, 1987, doi: 10.1109/TPAMI.1987.4767964. 